\title{
Inhibition of pleural metastasis of collecting duct carcinoma of the kidney by modified cytokine-induced killer cells: A case report and review of the literature
}

\author{
JINGYI LIU ${ }^{1 *}$, JUN SUI $^{1 *}$, ZHIWEI ZHANG ${ }^{1}$, XIUBAO REN ${ }^{3}$, LI LUAN $^{1}$, QISHENG YANG $^{2}$, \\ SONGHAI GU ${ }^{4}$, RUDOLF WANK ${ }^{4}$, BARBARA LAUMBACHER ${ }^{4}$ and XIN SONG ${ }^{1}$ \\ ${ }^{1}$ Cancer Biotherapy Center; ${ }^{2}$ Radiology Department, the Third Affiliated Hospital, Kunming Medical College, \\ Kunming; ${ }^{3}$ Department of Biotherapy, the Affiliated Cancer Hospital, Tianjin Medical University, \\ Tianjin, P.R. China; ${ }^{4}$ Immunotherapy Research Centre, D-80336 Munich, Germany
}

Received April 14, 2010; Accepted August 17, 2010

DOI: $10.3892 / \mathrm{ol} .2010 .168$

\begin{abstract}
Collecting duct carcinoma (CDC) or Bellini duct carcinoma of the kidney is a rare, but highly aggressive renal epithelial malignancy, with an extremely poor prognosis. Modified cytokine-induced killer (mCIK) cells were injected into the pleural cavity to treat pleural metastasis of CDC. The patient, a 33-year-old male, was admitted to hospital for further treatment for severe pleural metastasis of CDC. We cured the pleural metastasis through intrapleural infusion with mCIK cells. After receiving this innovative treatment, the patient exhibited a positive response: the cough, dyspnea, chest distress and thoracalgia were evidently relieved, while the pleural fluid became clear after exhibiting haematodes and its level decreased significantly. The patient achieved partial success. This novel immunotherapy method is a promising treatment for patients with refractory pleural metastasis.
\end{abstract}

\section{Introduction}

Collecting duct carcinoma (CDC) or Bellini duct carcinoma, which accounts for $1-3 \%$ of all renal neoplasms (1), is a rare variant of renal cell carcinoma (RCC) originating in the collecting duct epithelium and occurring more frequently in relatively young adults (2). Patients with CDC often require a more aggressive clinical course, have a poor long-term survival rate and, currently no standard treatment regimens exist (3). CDC and clear cell RCC typically present with a renal mass,

Correspondence to: Professor Xin Song, Cancer Biotherapy Center, the Third Affiliated Hospital, Kunming Medical College, Kunming 650118, P.R. China

E-mail: songxin68@yahoo.com.cn

*Contributed equally

Key words: collecting duct carcinoma, pleural metastasis, immunotherapy macroscopic hematuria and associated flank pain. However, the majority of patients with CDC show evidence of metastatic disease at the time of presentation (3). Pleural metastasis of CDC is resistant to chemotherapy and radiotherapy, while immunotherapy, especially adoptive immune cell treatment, becomes a promising treatment for pleural metastasis. The present study evaluated a patient with pleural metastasis of CDC who achieved partial success following intrapleural infusion with modified cytokine-induced killer (mCIK) cells.

\section{Case report}

A 33-year-old male with no significant past medical history was referred for urology review after a mass was found during a regular examination. A left radical nephrectomy was performed in December 2006 in the First Affiliated Hospital, Kunming Medical College, China. The pathology revealed CDC (Fig. 1) in the left kidney with no lymph node metastasis. After 1 year, the patient suffered from constant coughing, dyspnea, chest distress and thoracalgia. A chest X-Ray revealed a left pleural effusion. The pleural effusion recurred 1 month after B-ultrasound-guided therapeutic thoracentesis. For further treatment, the patient was referred to our institution on May 5th, 2008.

A physical examination revealed a dull sound on percussion and the breath sounds disappeared below the sixth left rib. A computed tomography (CT) scan of the chest, abdomen and pelvis revealed no local recurrence and no lymph node metastasis, although severe pleural metastasis was noted. The examination results revealed that the left pleural had thickened, the pleural effusion was partly encapsulated and there was left pulmonary atelectasis (Fig. 3A). An ultrasoundguided aspiration revealed haematodes fluid. The results of the laboratory examinations were insignificant. Haematology showed a haemoglobin level of $171 \mathrm{~g} / 1$ (120-160), a white cell count of $11.72 \times 10^{9 / 1}(4-10)$ and a platelet count of $236 \times 10^{9 / 1}$ (100-300).

To alleviate pleural effusion and dyspnea, the patient was treated with a mCIK cell intrapleural infusion. Mononuclear cells were obtained aseptically from blood and infused back 

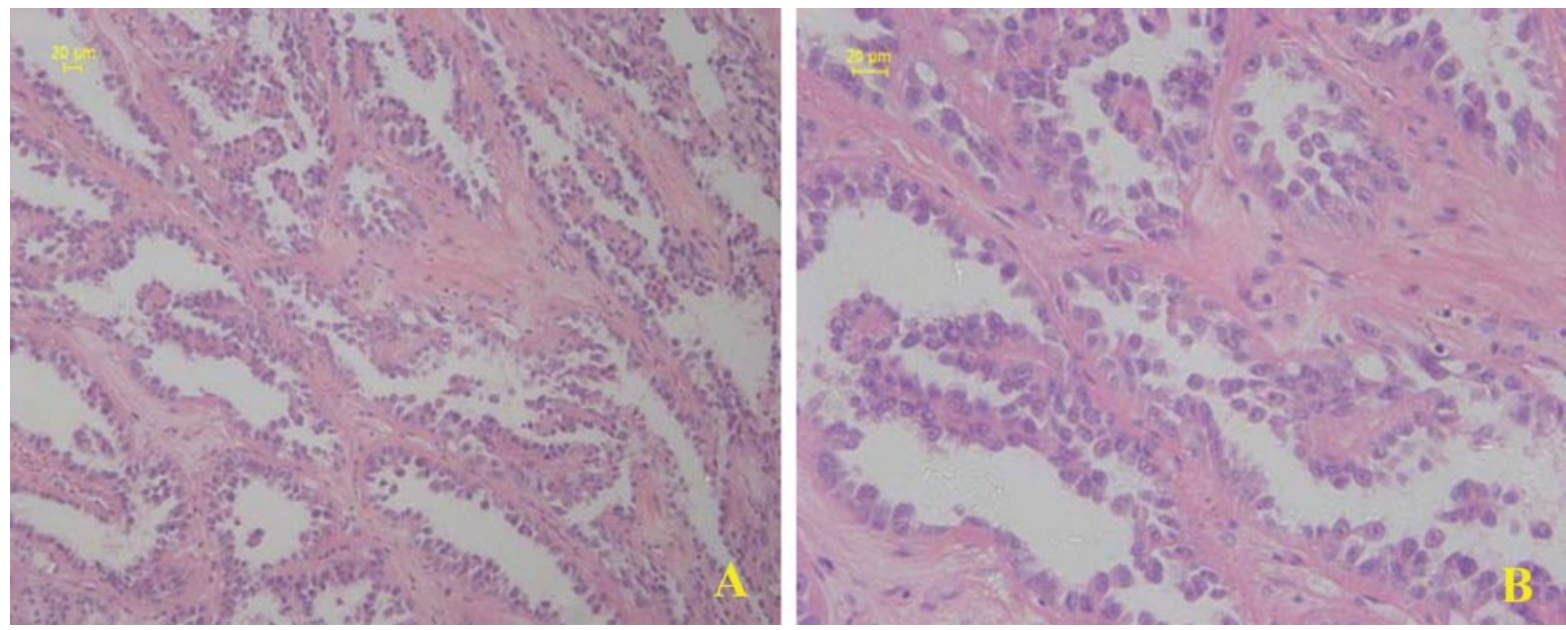

Figure 1. Histological features of CDC in a representative section. (A) Typical irregular angulated tubular architecture associated with striking stromal desmoplasia. (B) High-powered view of CDC showing cancer cells with large pleomorphic nuclei and eosinophilic cytoplasm. Pathological tissue section No. 15397. Scale bars, $20 \mu \mathrm{m}$.
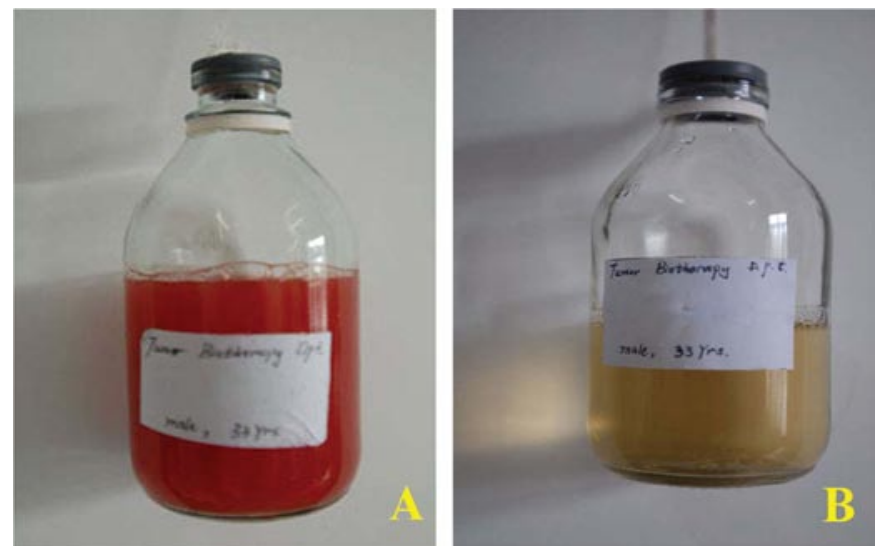

Figure 2. Pleural fluid change. (A) Pre-therapy: haematodes fluid (B) Following the second treatment cycle: clear fluid.

to the patient through intrapleural infusion, following in vitro culture of the mCIK cells.

The mCIK cells were cascade-primed immune cells (CAPRI) generated using a procedure to enhance cytokineinduced killer cells (4), with modification. Briefly, using a blood cell separator (Spectra, Gambro BCT, USA), leukapheresis was conducted to obtain peripheral blood mononuclear cells (PBMCs). Half of the mononuclear cells were resuspended in culture flasks coated with anti-CD3 antibody (BD Pharmingen, NJ, USA), and incubated at $37^{\circ} \mathrm{C}$ in a humidified atmosphere of $5 \% \mathrm{CO}_{2}$. After $3 \mathrm{~h}$, a mixture of $700 \mathrm{U} / \mathrm{ml}$ interleukin (IL)-2, $800 \mathrm{U} / \mathrm{ml} \mathrm{IL-18}$ and 2,000 U/ml interferon (IFN) $-\gamma$ (all from R\&D Systems, MN, USA) were added. After another $3 \mathrm{~h}$, the remaining mononuclear cells were added. After $24 \mathrm{~h}$, the cells were transferred to a larger culture flask for expansion. IL-2, IL-18 and IFN- $\gamma$ were added every 2 days. After incubation for 7 days, the activated immune cells were harvested and preserved in liquid nitrogen. The cells were defrosted, resuspended in phosphate-buffered saline and freshly transfused to the patient when required.

In every cycle, the patient received mCIK cells at a dose of 100 million $(40 \mathrm{ml})$ once a day for 5 days, through intrapleural infusion. Following the initial cycle of treatment, the coughing disappeared and symptoms including dyspnea, chest distress and thoracalgia were relieved. In order to receive further treatment, the patient returned to the department for the second cycle of therapy on July 18, 2008. An ultrasound-guided aspiration revealed $600 \mathrm{ml}$ of clear fluid (Fig. 2B). A CT scan (Fig. 3B) revealed that the left pulmonary had re-expanded and the level of pleural fluid had decreased. After the third cycle of therapy, the dyspnea was evidently relieved and the chest distress and thoracalgia were relieved completely. The chest X-Ray (Fig. 4A) revealed a great reduction in the level of pleural fluid compared to the initial examination (Fig. 4B).

The patient had a fever $\left(\sim 38^{\circ} \mathrm{C}\right)$ after intrapleural infusion, but recovered 2 days later. No other adverse effects were noted during therapy.

The evaluation of efficacy was based on the relief of symptoms and absence or reduction of pleural fluid $(5,6)$. Patients with symptomatic improvement and no detectable fluid on the roentgenogram prior to discharge are deemed a success. Those with symptomatic improvement, but residual fluid in the costophrenic angle, with no tendency for increase, are considered to be a partial success. Patients whose pleural effusion recurred or who had no symptomatic improvement, are deemed unsuccessful. Based on these criteria, the patient received mCIK cells and achieved partial success.

\section{Discussion}

The rare renal carcinoma type $\mathrm{CDC}$, first reported by Mancilla-Jimenz (7), exhibits highly aggressive behavior (8). Although radical surgery is the basic type of therapy and different protocols have been applied, the challenge involves the identification of novel therapeutic modalities (9). No standard protocols have been established for the treatment of CDC. Numerous agents have been reported over the last few decades as being effective by intrapleural injection in the suppression of recurrent malignant pleural effusion (10). Intrapleural injection by mCIKs is a novel therapeutic strategy for the treatment of malignant pleural effusions. We evaluated 

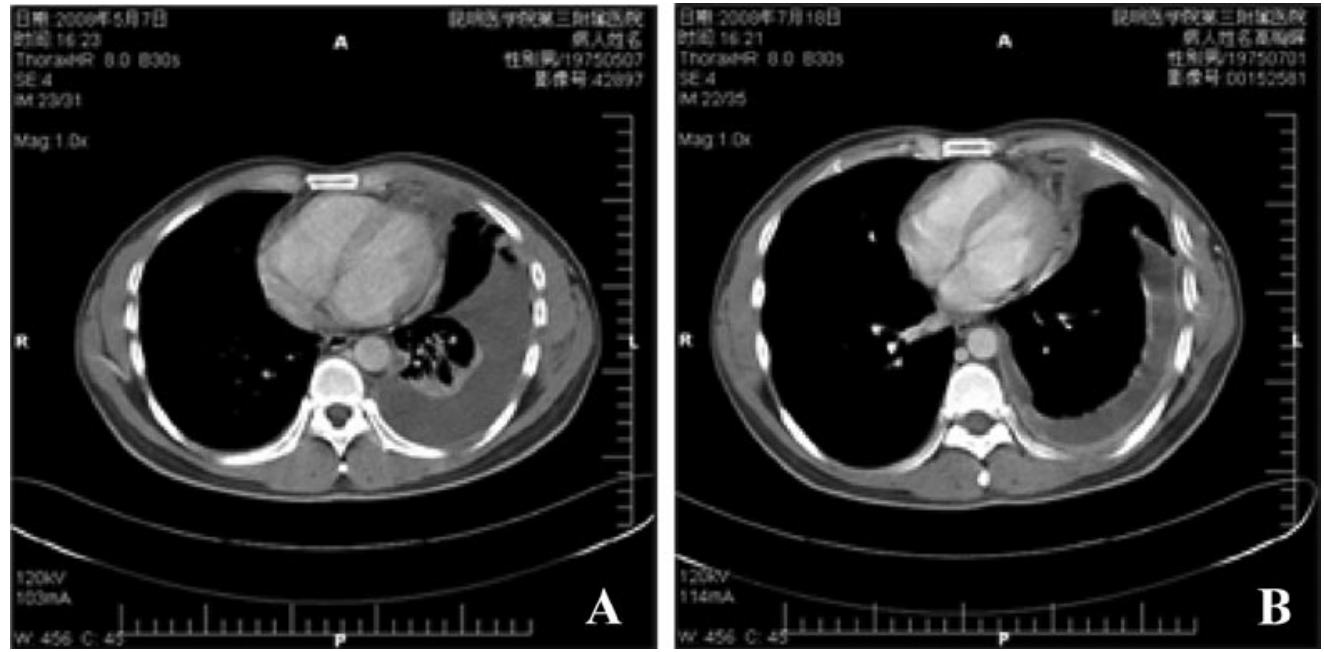

Figure 3. CT scan. (A) Pre-therapy: pulmonary atelectasis. (B) After the second cycle of treatment pulmonary re-expansion is evident.
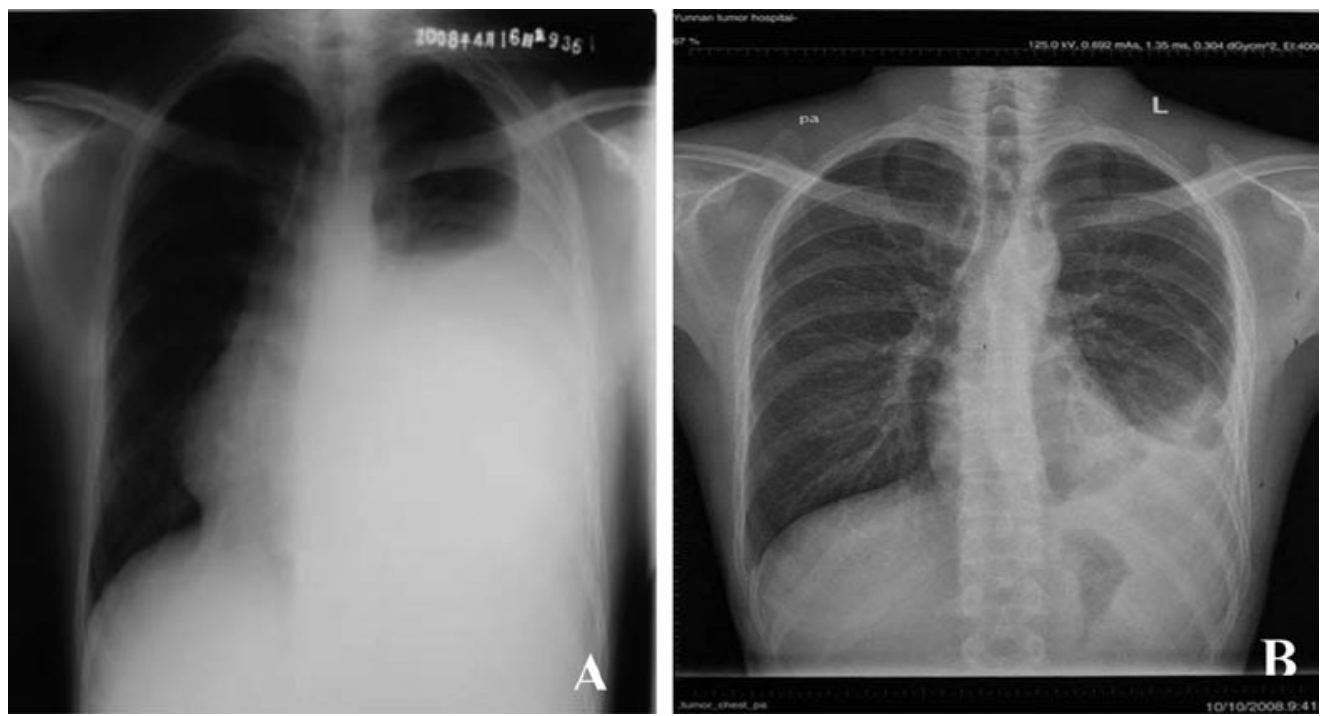

Figure 4. Alteration of chest X-Ray. (A) Pre-therapy reveals a large amount of pleural fluid in the left thoracic cavity. (B) After the third cycle of treatment, the level of pleural fluid decreased significantly.

this case due to the efficacy of this novel immunotherapy method in the supression of pleural metastasis of CDC.

mCIK cells are a cluster of cascade-primed immune cells, including mainly $\mathrm{CD}^{+}{ }^{+} \mathrm{CD} 56^{+}$and $\mathrm{CD} 3{ }^{+} \mathrm{CD} 8^{+} \mathrm{T}$ cells. PBMCs were stimulated in vitro with $\mathrm{CD} 3$-activated $\mathrm{T}$ lymphocytes in order to increase the expression of HLA molecules and costimulatory molecules. In particular, the antigen-presenting cells in the molecules express tumor peptides after stimulation. Freshly added PBMCs were primed by the stimulated antigen-presenting cells and immune cytokines (IL-18, IFN- $\gamma$ and IL-2) and matured to helper $\mathrm{T}$ and cytotoxic $\mathrm{T}$ cells. IL-18 induces enhanced CD8 + T-cell proliferation (11). Yamada et al found that IL-18 immunotherapy decreased the number of macroscopic pulmonary metastases (12). IFN- $\gamma$ is the hallmark cytokine of Th1 cells and stimulates anti-tumor immunity (13). IL-2 stimulates the growth, differentiation and survival of antigen-selected cytotoxic $\mathrm{T}$ cells and enhances anti-tumor immunity (14). The mCIK cells proliferated rapidly and showed anti-tumor effects on various types of cancer.
We treated pleural metastasis of the patient with this novel immunotherapy method. Following the initial cycle of treatment, the symptoms including coughing, chest distress and thoracalgia were relieved. After the second cycle of treatment, we found that pleural fluid previously exhibiting haematodes had become clear. Although the pleural effusion remained, pulmonary re-expansion was evident (Fig. 3B). After the third cycle of treatment was completed, the levels of pleural fluid had decreased significantly (Fig. 4B). According to the previous criteria, the patient achieved partial success.

Various agents have been instilled into the pleural space to treat malignant pleural effusions and agents, such as talc, tetracycline and bleomycin, creating a chemical pleuritis $(10,15)$. However, when we treated the patient with mCIK cells, overt pleuritis was not observed, only a transitory fever, suggesting the safety of this method for the treatment of pleural metastasis.

The intrapleural infusion of activated immune cells causes the accumulation of effector cells and significantly decreases 
the number of tumor cells. The precise mechanisms of eradication of tumor cells by the adoptive transfer of activated killer cells have yet to be elucidated, but this transfer may be mediated mainly by the immune responses of cytotoxic killer $\mathrm{T}$ cells. Intrapleural infusion is an innovative method aimed at curing pleural metastasis with activated immune cells. Thus, the efficacy and mechanisms of this exploratory treatment require further study.

In conclusion, to control malignant pleural fluid, drainage and intrapleural chemotherapy are applied in the majority of cases of pleural metastasis. However, successful supression of pleural fluid is difficult and always recurs in advanced cases. This study suggested that mCIK cell intrapleural infusion is a candidate for the therapy of pleural metastasis with refractory pleural fluid.

\section{Acknowledgements}

This study was supported by grants from the National Natural Science Foundation of China (Grant No. 30671904, 30760014 and 81060185), the New Century Excellent Talent Supporting Project of Ministry of Education of China (Grant No. NCET08-0923), the Cultivation Program for Reserve Talent of Middle-Young Aged Academic and Technology Leader of Yunnan Province (Grant No. 2006Y01-12), the Key Program of Science and Technology Project of Kunming (Grant No. 07S060202), the Yunnan Provincial Applied Basic Research Program (Grant No. 2007C0022R, 2007C0025R and 2007C0023R), the Science and Technology Program of Yunnan Province (Grant No. 2007C009Z), the Yunnan Provincial Key Program for Applied Basic Research (Grant No. 2008CC006), the Innovation talent Team Program for Prevention and Treatment of High Incidence Lung Cancer of Yunnan Province (Grant No. 20080C014), and the International Technology Cooperation Project of Scientific and Technological Innovation Program of Yunnan Province Science and Technology Agency (Grant No. 2009AC016).

\section{References}

1. Dimopoulos MA, Logothetis CJ, Markowitz A, et al: Collecting duct carcinoma of the kidney. Br J Urol 71: 388-391, 1993.

2. Méjean A, Rouprêt M, Larousserie F, et al: Is there a place for radical nephrectomy in the presence of metastatic collecting duct (Bellini) carcinoma? J Urol 169: 1287-1290, 2003.

3. Milowsky MI, Rosmarin A, Tickoo SK, Papanicolaou N and Nanus DM: Active chemotherapy for collecting duct carcinoma of the kidney: a case report and review of the literature. Cancer 94: 111-116, 2002.

4. Shi M, Zhang B, Tang ZR, et al: Autologous cytokine-induced killer cell therapy in clinical trial phase is safe in patients with primary hepatocellular carcinoma. World J Gastroenterol 10: 1146-1151, 2004.

5. Millar JW, Hunter AM and Horne NW: Intrapleural immunotherapy with Corynebacterium parvum in recurrent malignant pleural effusions. Thorax 35: 856-858, 1980.

6. Schulze M, Boehle AS, Kurdow R, et al: Effective treatment of malignant pleural effusion by minimal invasive thoracic surgery: thoracoscopic talc pleurodesis and pleuroperitoneal shunts in 101 patients. Ann Thorac Surg 71: 1809-1812, 2001.

7. Mancilla-Jimenez R, Stanley RJ and Blath RA: Papillary renal cell carcinoma. A clinical, radiologic, and pathologic study of 34 cases. Cancer 38: 2469-2480, 1976.

8. Anjum MI, Ting PY, Shrotri N, Randall B and Mufti GR: An unusual case of Bellini duct carcinoma. Int Urol Nephrol 28: 695-698, 1996.

9. Ansari J, Fatima A, Chaudhri S, et al: Sorafenib induces therapeutic response in a patient with metastatic collecting duct carcinoma of kidney. Onkologie 32: 44-46, 2009.

10. Musani AI: Treatment options for malignant pleural effusion. Curr Opin Pulm Med 15: 380-387, 2009.

11. Balkow S, Loser K, Krummen M, et al: Dendritic cell activation by combined exposure to anti-CD40 plus interleukin (IL)-12 and IL-18 efficiently stimulates anti-tumor immunity. Exp Dermatol 18: 78-87, 2009.

12. Yamada N, Hata M, Ohyama $\mathrm{H}$, et al: Immunotherapy with interleukin-18 in combination with preoperative chemotherapy with ifosfamide effectively inhibits postoperative progression of pulmonary metastases in a mouse osteosarcoma model. Tumour Biol 30: 176-184, 2009.

13. Olioso P, Giancola R, Di Riti M, et al: Immunotherapy with cytokine induced killer cells in solid and hematopoietic tumours: a pilot clinical trial. Hematol Oncol 27: 130-139, 2009.

14. McDermott DF: Immunotherapy of metastatic renal cell carcinoma. Cancer 115: 2298-2305, 2009.

15. Hausheer FH and Yarbro JW: Diagnosis and treatment of the malignant pleural effusion. Semin Oncol 12: 54-75, 1985. 